\title{
EMPREGO DA ANÁLISE DE CAOS NA CARACTERIZAÇÃO DE REGIMES FLUIDIZADOS PARA PARTÍCULAS DO GRUPO A DA CLASSIFICAÇÃO GELDART
}

\author{
W. H. PRIETO ${ }^{1 *}$, M. A. CREMASCO ${ }^{1}$ \\ ${ }^{1}$ Universidade Estadual de Campinas, Faculdade de Engenharia Química, Departamento de \\ Engenharia de Processos \\ *e-mail: wesley@feq.unicamp.br
}

\begin{abstract}
RESUMO
Devido à complexa dinâmica apresentada pelos leitos fluidizados se faz necessário o desenvolvimento de novas de técnicas de análise de sinais que representem, com maior fidelidade, as características reais destes processos, principalmente quando técnicas convencionais não são apropriadas para a caracterização de regimes de fluidização. É justamente neste cenário que surge a análise do caos determinístico como alternativa às metodologias clássicas empregadas. O presente trabalho objetiva aplicar a teoria do caos a sinais de variação de pressão, associando os invariantes caóticos, $\mathrm{K}$ e $\mathrm{D}$, a regimes fluidodinâmicos de um leito fluidizado gás-partícula. Para tanto, utilizou-se uma coluna de acrílico (0,1 m de diâmetro) e ar na fluidização de partículas de catalisador FCC e microesferas de vidro, ambas pertencentes ao grupo A da classificação Geldart. Na obtenção dos sinais de pressão, foram empregados transdutores diferenciais de pressão a taxas de $1.000 \mathrm{~Hz}$. Para ambas as partículas, notou-se acréscimo nos valores de K e D a medida em que a velocidade superficial do ar aumenta, alcançando valores máximos de tais invariantes na transição do leito fixo para a condição de mínima fluidização. A complexidade do sistema decai quando se atinge o regime pistonado. Como decorrência, constata-se que tanto a entropia de Kolmogorov quanto a dimensão de correlação podem ser empregadas como parâmetros de caracterização de regimes em sistemas fluidizados.
\end{abstract}

\section{INTRODUÇÃO}

Devido à complexidade da interação gás-partícula, presente em sistemas fluidizados, surge a necessidade do desenvolvimento de técnicas de análise que, com maior fidelidade, possam representar as características deste fenômeno. Os leitos fluidizados são aplicados para inúmeras finalidades, entre elas destacam-se: secagem, mistura, sínteses catalíticas, granulação, recobrimento e muitas outras (CASTILHO, 2011; CREMASCO, 2012). O princípio básico da fluidização é o movimento vertical e/ou circulatório de partículas sob ação de um fluido ascendente. $\mathrm{O}$ grande interesse por esta tecnologia, justificado por sua ampla aplicação industrial, se iniciou na década de 1940 com o advento do craqueamento catalítico de petróleo e, a partir de então, várias propostas foram feitas intuindo o desenvolvimento de uma metodologia adequada para análise e dimensionamento deste tipo de sistemas.

Um dos métodos consagrados para o estudo de séries temporais é a análise espectral (domínio da frequência) (HONG et al., 1990; KAGE et al., 1991; BAI et al., 1999; JOHNSSON et al., 2000; TRNKA et al., 2000; BROWN E BRUE, 2001; SASIC et al., 2007; MARTÍN et al., 2011). Esta metodologia normalmente utiliza a 
Transformada de Fourier e visa a obtenção de frequências dominantes, características a cada um dos regimes fluidodinâmicos da fluidização, (KAGE et al., 1991) presentes no espectro de potências. $\mathrm{Na}$ maioria dos processos fluidomecânicos nota-se que a frequência dominante não pode ser verificada, obrigando a formulação de hipóteses simplificadoras, que imprimem erros à análise, para contornar esta problemática. É neste cenário que surge a análise do caos como ferramenta alternativa à metodologia espectral, pois sua aplicação dispensa a necessidade de um espectro de potências bem comportado.

A teoria do caos utiliza-se da reconstrução de atratores em um espaço de fases e do estudo dos graus de previsibilidade e de sensibilidade de um sistema à perturbação na investigação de sistemas dinâmicos. Resumidamente, a análise de caos é aplicada a processos dissipativos, cuja evolução temporal é não linear, aperiódica a longo prazo, determinístico e extremamente sensível às condições iniciais (SAVI, 2004). Um fenômeno que possua estas características certamente apresentará, durante sua evolução temporal, uma contração no volume do espaço de fases e suas linhas de fluxo dependerão das condições iniciais (RUELLE E TAKENS, 1971) definindo o chamado atrator estranho ou atrator caótico. A utilização da análise de caos aplicada aos processos de engenharia teve início com o trabalho pioneiro de Stringer (1989).

Qualquer que seja o fenômeno físico estudado, o procedimento de análise por meio da teoria caótica determinística é o mesmo. Uma vez identificado o problema a ser estudado, deve-se obter uma série temporal relativa a uma variável de interesse que represente $\mathrm{o}$ fenômeno. Esta evolução temporal pode ser medida a partir de uma instrumentação adequada ou simulada teoricamente por meio de equações. Depois de obtida a série, passa-se à reconstrução do atrator em um espaço de fases seguido do cálculo, a partir da topologia do atrator, dos invariantes caóticos.

O presente trabalho objetiva aplicar a abordagem do caos determinístico a sinais experimentais de variação de pressão total de um leito fluidizado gás-partícula, associando os invariantes caóticos, entropia de Kolmogorov e dimensão de correlação, às regiões fluidodinâmicas utilizando partículas A da classificação Geldart.

\section{MATERIAIS E MÉTODOS}

A unidade experimental utilizada neste trabalho está instalada no Laboratório de Processos em Meios Porosos (LPMP) da Faculdade de Engenharia Química da Universidade Estadual de Campinas. $\mathrm{Na}$ Figura 1, observa-se a unidade experimental completa. O ponto A indica a coluna de fluidização, B aponta o ciclone, $\mathrm{C}$ indica o distribuidor e D o painel em que foi instalado o sistema de aquisição de dados. Todo o aparato foi fixado em um suporte metálico.

Figura 1 - Unidade experimental de fluidização.

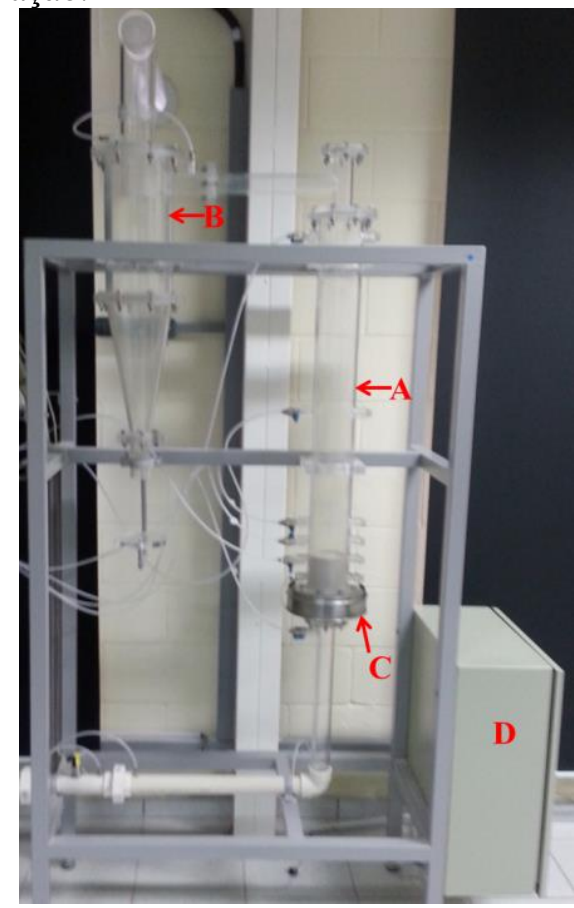


A coluna de fluidização foi confeccionada em material acrílico, com diâmetro de $0,10 \mathrm{~m}$ e altura $1,0 \mathrm{~m}$. A vazão de ar (variável) foi fornecida por um soprador, de vazão máxima $6,5 \times 10^{-2} \mathrm{~m}^{3} / \mathrm{s}$ e pressão de 35,3 $\mathrm{kPa}$. O distribuidor $(\mathrm{Dd}=0,1 \mathrm{~m})$ instalado apresenta orifícios de $2,7 \times 10^{-3} \mathrm{~m}$ de diâmetro e organização triangular com espaçamento entre orifícios de $0,75 \mathrm{~cm}$. Utilizou-se uma tela de 400 mesh para não permitir a passagem das partículas pelo distribuidor. Foi elaborada uma curva característica de queda de pressão do distribuidor para, posteriormente, descontar da perda total do leito.

A evolução temporal da pressão foi obtida por meio de transdutor diferencial de pressão tipo Novus NP8OOH, com uma tomada instalada logo abaixo do distribuidor e outra no topo da coluna, obtendo-se a variação total de pressão do leito. A frequência e o tempo ótimo de amostragem foram respectivamente de $1.000 \mathrm{~Hz}$ e 40 segundos. O sistema de aquisição utilizado foi do tipo National Instruments N19403 e o software NI LabView 2013. A filtragem dos dados foi realizada utilizando um filtro do tipo passa-alta de $10 \mathrm{~Hz}$. Com o objetivo de eliminar ruídos da rede elétrica, instalaram-se duas baterias seladas de $12 \mathrm{~V}$.

As curvas de fluidização foram obtidas de forma descendente da vazão de ar. Todos os experimentos foram realizados em triplicata. As fases particuladas analisadas foram o FCC (fluid catalytic cracking) e microesferas de vidro. A fase gasosa foi o ar à temperatura de $25^{\circ} \mathrm{C}$. A Tabela 1 lista as propriedades dos materiais.

Para o cálculo dos invariantes caóticos, utilizou-se o software Basic Resolution of Chaos (BRChaos), implementado por Moura (2013). Os atratores foram reconstruídos com 40.000 pontos e considerou-se tempo de atraso $(\theta)$ igual ao tempo de amostragem (SUGIHARA e MAY, 1990; YU et al., 2013), portanto, $\theta=0,001 \mathrm{~s}$.
Tabela 1 - Propriedades das fases sólidas e gasosa.

\begin{tabular}{|c|c|c|c|}
\hline Fase Sólida & FCC & $\begin{array}{c}\text { Microesferas } \\
\text { de Vidro }\end{array}$ & \\
\hline $\mathrm{d}_{\mathrm{p}}$ & $79,65 \times 10^{-6}$ & $64,0 \times 10^{-6}$ & $\mathrm{~m}$ \\
\hline$\rho_{\mathrm{p}}$ & 901,7 & 2500,0 & $\mathrm{~kg} / \mathrm{m}^{3}$ \\
\hline Classificação & Geldart A & Geldart A & \\
\hline Massa & 0,4 & 0,4 & $\mathrm{~kg}$ \\
\hline Altura & 0,07 & 0,07 & $\mathrm{~m}$ \\
\hline Fase Gasosa & $\mathrm{Ar}$ & \multirow{3}{*}{\multicolumn{2}{|c|}{$\begin{array}{c}{ }^{\circ} \mathrm{C} \\
\mathrm{kg} / \mathrm{m}^{3}\end{array}$}} \\
\hline Temperatura & 25 & & \\
\hline$\rho$ & 1,184 & & \\
\hline
\end{tabular}

\section{RESULTADOS E DISCUSSÕES}

Os resultados obtidos foram divididos em duas partes, sendo a curva de fluidização e a identificação dos regimes de fluidização utilizando a teoria do caos. Em cada uma destas etapas incluiu-se um comparativo entre o FCC e as microesferas de vidro.

\subsection{Curvas de fluidização}

A Fig. 2 representa o perfil de queda de pressão $(-\Delta \mathrm{P})$ com $\mathrm{o}$ aumento da velocidade superficial do ar para um carregamento de $0,4 \mathrm{~kg}$ de partículas catalisador FCC.

Cada ponto da curva corresponde à média, em triplicata, de 40.000 pontos obtidos pelos transdutores diferenciais de pressão. Estimou-se a velocidade de mínima fluidização por meio da intersecção das retas interpoladas para a região linear de leito fixo e para a região de $-\Delta \mathrm{P}$ constante após a mínima fluidização. O ponto de mínima fluidização esta indicado na Figura 2, sendo o valor encontrado para a $\mathrm{U}_{\mathrm{mf}}=0,26 \mathrm{~m} / \mathrm{s}$ com respectiva $\left(-\Delta \mathrm{P}_{\mathrm{mf}}\right)=514 \mathrm{~Pa}$.

As regiões do gráfico identificadas por (I), (II) e (III) são os pontos em que ocorreram as transições de regime. (I) representa o leito fixo, região em que se verifica o aumento, praticamente linear e bem acentuado, da queda de pressão. Esta é a região em que o leito oferece maior resistência à injeção de ar, 
notando-se que a posição das partículas sofre pequenas alterações com a passagem do fluido e na medida em que o a velocidade do gás aumenta, aproximando-se da mínima fluidização, a movimentação do leito fica mais vigorosa.

Depois de alcançada a mínima fluidização, notou-se a formação de bolhas e, portanto, a migração para o regime borbulhante (Região (II)). A última região, caracterizada pelo aumento da queda de pressão, acima da mínima fluidização, corresponde ao regime pistonado. Para o FCC esta transição ocorreu após a razão $\mathrm{U} / \mathrm{U}_{\mathrm{mf}} \approx$ 1,20. É importante frisar que a identificação das fases de transição foi realizada por meio de observação direta das características do leito durante o processo.

Na Figura 3, apresenta-se a curva de fluidização para partículas de microesferas de vidro. Cada um dos pontos do gráfico corresponde à média, em triplicata, de 40.000 pontos obtidos pelos transdutores diferenciais de pressão. Ar foi utilizado como fluido de trabalho e a coluna foi carregada com $0,4 \mathrm{~kg}$ de microesferas de vidro.

Figura 2 - Curva de fluidização das partículas de FCC.

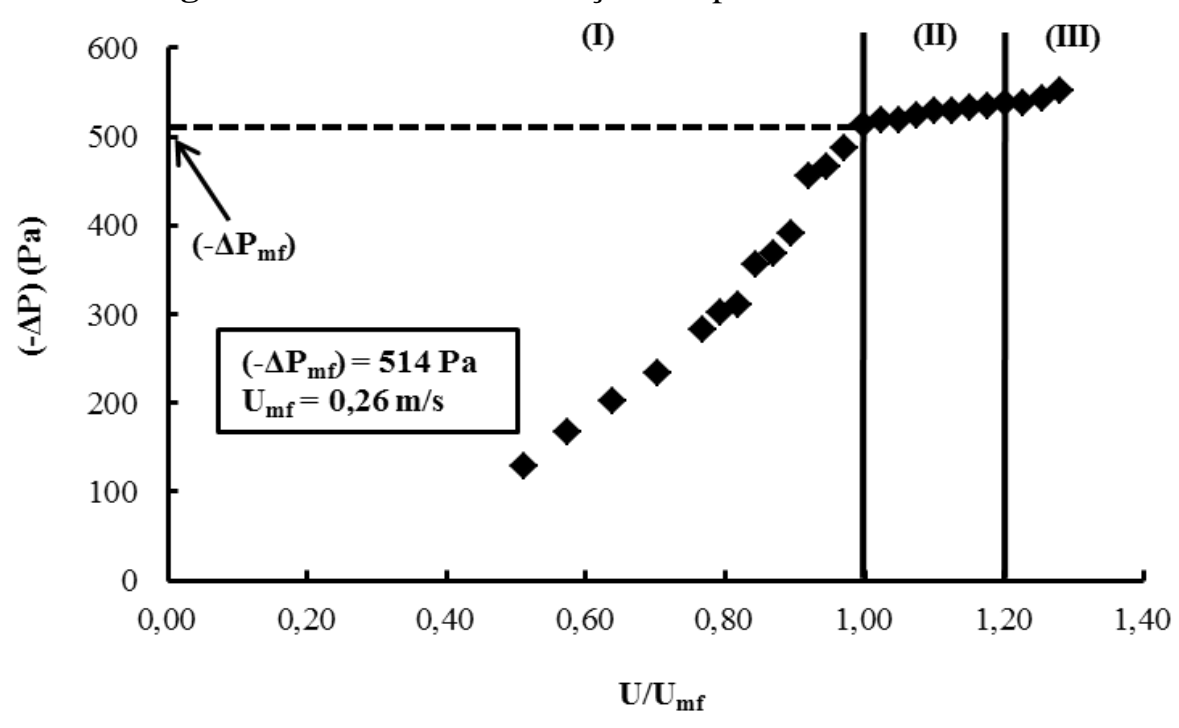

Figura 3 - Curva de fluidização das partículas de microesferas de vidro.

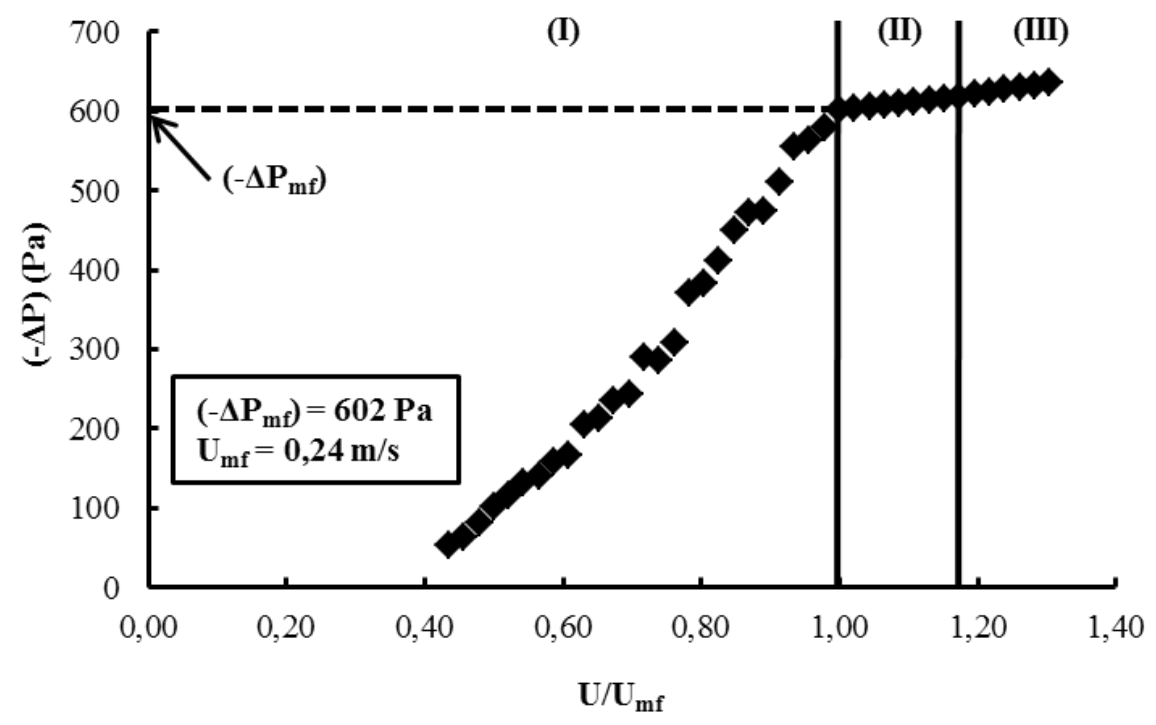


Análogo ao que foi feito para o FCC, a velocidade de mínima fluidização foi obtida por meio da intersecção das retas interpoladas para a região linear de leito fixo e para a região de $-\Delta \mathrm{P}$ constante após a mínima fluidização. A seta indicativa na Fig. 3 aponta a mínima fluidização, sendo os valores encontrados para a $\mathrm{U}_{\mathrm{mf}}$ e $\left(-\Delta \mathrm{P}_{\mathrm{mf}}\right)$ de respectivamente $0,24 \mathrm{~m} / \mathrm{s}, 602 \mathrm{~Pa}$. Similar ao discutido anteriormente, (I), (II) e (III) são os pontos em que ocorreram as transições de regime, sendo (I) o leito fixo, (II) o regime borbulhante e (III) pistonado. Para as microesferas de vidro a transição borbulhante/pistonado foi observada para $\mathrm{U} / \mathrm{U}_{\mathrm{mf}} \approx 1,15$.

Em ambos os casos a compactação dos leitos de microesferas de vidro e FCC, devido ao pequeno diâmetro das partículas e das características pulverulentas dos materiais, faz com que o a queda de pressão, com a variação da velocidade do ar, seja suave, conferindo a esta região um perfil característico pouco inclinado. Outro impacto das propriedades físicas dos dois materiais é a rápida transição do regime borbulhante para o regime pistonado. Um comparativo entre os dois processos permite verificar que as partículas de FCC necessitam de maior velocidade do gás para promover a fluidização. Esta diferença entre as partículas é esperada, pois devido à alta compactação do leito de FCC, são necessárias maiores velocidades de gás para iniciar a fluidização.

\subsection{Aplicação da teoria do caos na identificação dos regimes fluidodinâmicos}

As Figuras 4 e 5 exibem as séries temporais e a reconstrução de atratores gerados para o FCC e para as microesferas de vidro respectivamente, para diferentes velocidades do gás. Todos os atratores foram reconstruídos com dimensão de imersão igual a 20 e com 40.000 pontos.

Evidencia-se a presença de caos mesmo para as velocidades abaixo da mínima fluidização. A explicação para este fenômeno é que nas proximidades da mínima fluidização o leito apresenta padrões vibratórios alternados, principalmente junto ao distribuidor, que fazem com que ocorram grandes variações de pressão e, por sua vez, caracterizam uma região altamente imprevisível e sensível às condições de processo. Outra verificação é que nas proximidades da transição do regime borbulhante/pistonado o sistema tende a se tornar menos caótico. Para elucidar adequadamente estes fenômenos, é importante recorrer aos invariantes entropia de Kolmogorov (K) e dimensão de correlação (D).

Os perfis dos valores de $\mathrm{K}$ encontrados podem ser observados nas Figura 6 e 7, em que ocorre o aumento de $\mathrm{K}$ com a velocidade do gás, atingindo o valor máximo nas proximidades da mínima fluidização, pois os caminhos preferenciais, antes formados no leito empacotado, se desfazem permitindo a passagem do gás. Diferentemente do que foi verificado na curva de fluidização, após a $U_{m f}$ observa-se um regime de transição e, em seguida, devido à baixa altura do leito, verifica-se o regime tipo pistonado. As letras nos gráficos indicam as regiões identificadas com a análise de caos, sendo (EL) a expansão do leito, (T) transição e (P) leito pistonado. De maneira similar, van den Bleek e Schouten (1993a) relataram que nas proximidades da transição para o leito fluidizado o valor da entropia de Kolmogorov atinge seu máximo. Llop et al. (2012) observaram uma brusca queda da entropia de Kolmogorov quando ocorre transição para o regime pistonado.

Comparando as duas figuras, nota-se que os valores de entropia de Kolmogorov para as microesferas de vidro são substancialmente menores do que para o FCC, isso porque a massa específica e esfericidade das esferas de vidro faz com que o leito se acomode com mais facilidade, sendo sua dinâmica menos complexa do que para o FCC. 
Figura 4-Atratores gerados para diferentes velocidades do gás - FCC.

$$
\mathrm{U}=\mathbf{0 , 1 7} \mathrm{m} / \mathrm{s} \text { (leito fixo) }
$$
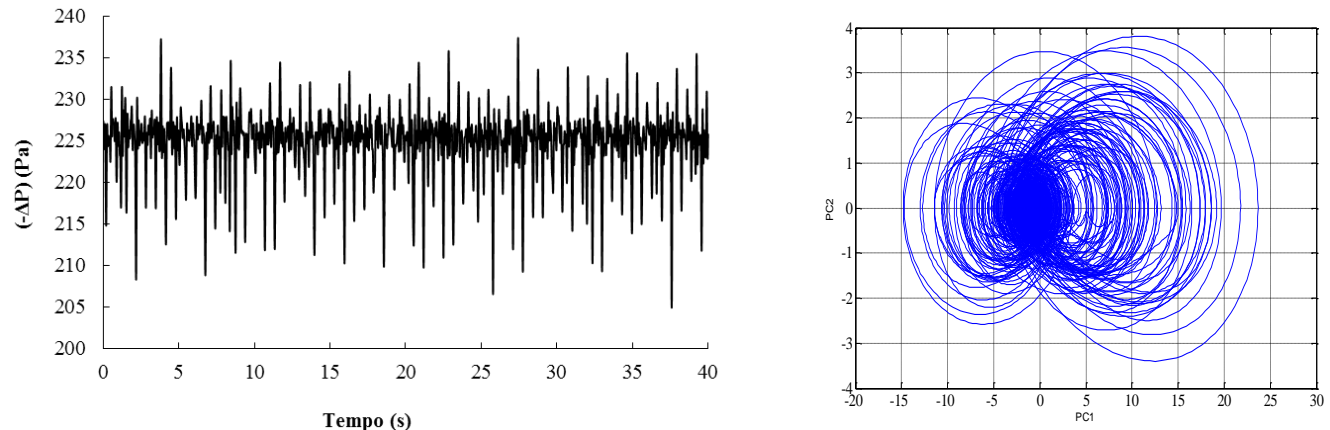

$$
\mathrm{U}=\mathbf{0 , 2 6} \mathrm{m} / \mathrm{s} \text { (mínima fluidização) }
$$
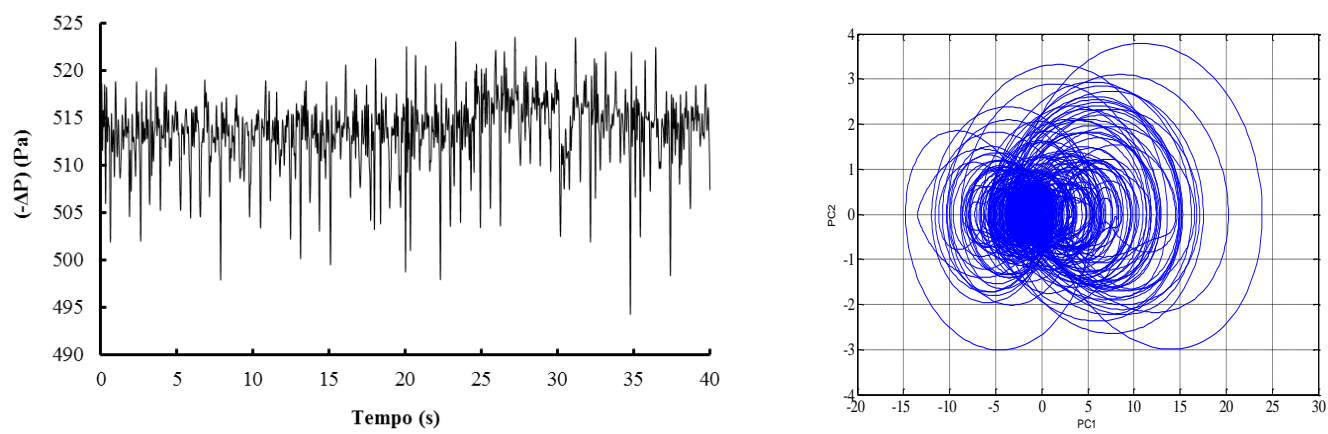

$\mathrm{U}=0,25 \mathrm{~m} / \mathrm{s}$ (regime borbulhante)
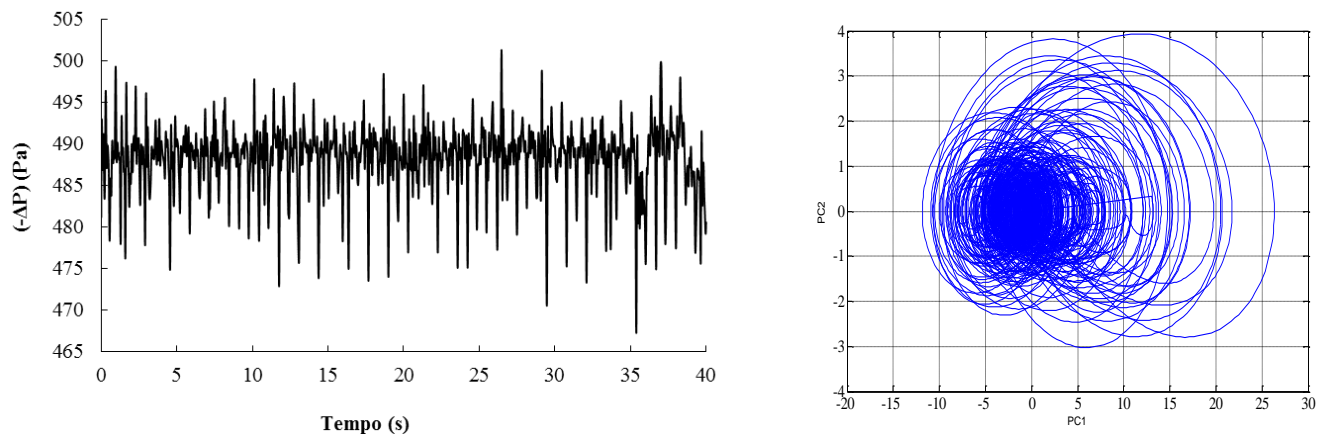

\section{$\mathrm{U}=\mathbf{0 , 3 3} \mathrm{m} / \mathrm{s}$ (pistonado)}
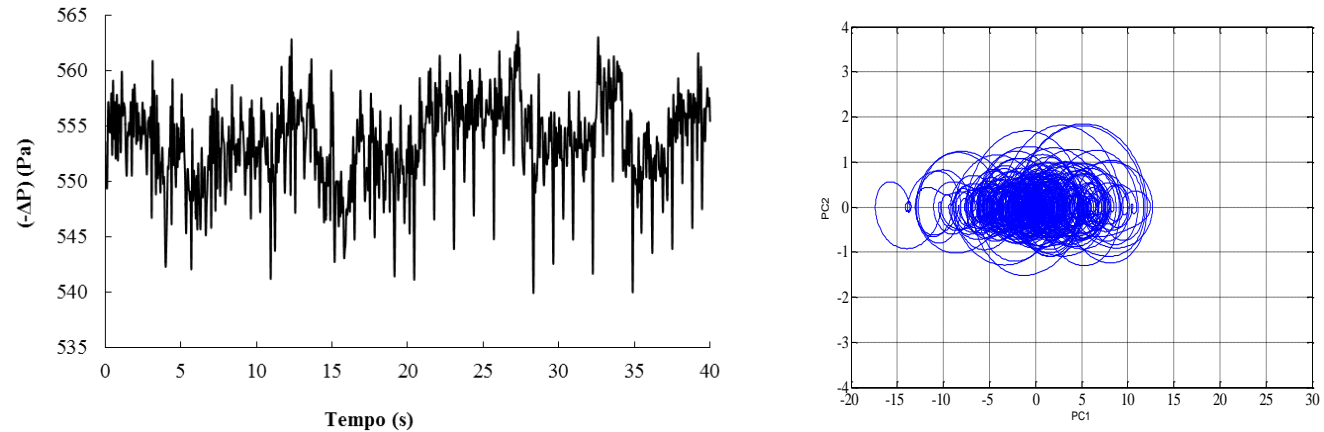
Figura 5 - Atratores gerados para diferentes velocidades do gás - Microesferas de vidro.

\section{$\mathrm{U}=\mathbf{0 , 1 5} \mathrm{m} / \mathrm{s}$ (leito fixo)}
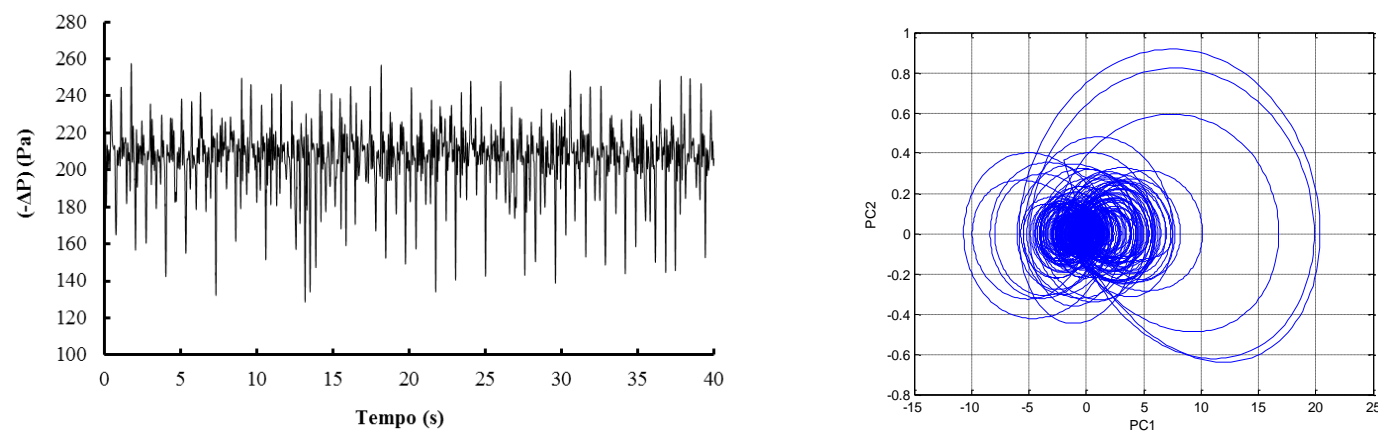

\section{$\mathrm{U}=\mathbf{0 , 2 4} \mathrm{m} / \mathrm{s}$ (mínima fluidização)}
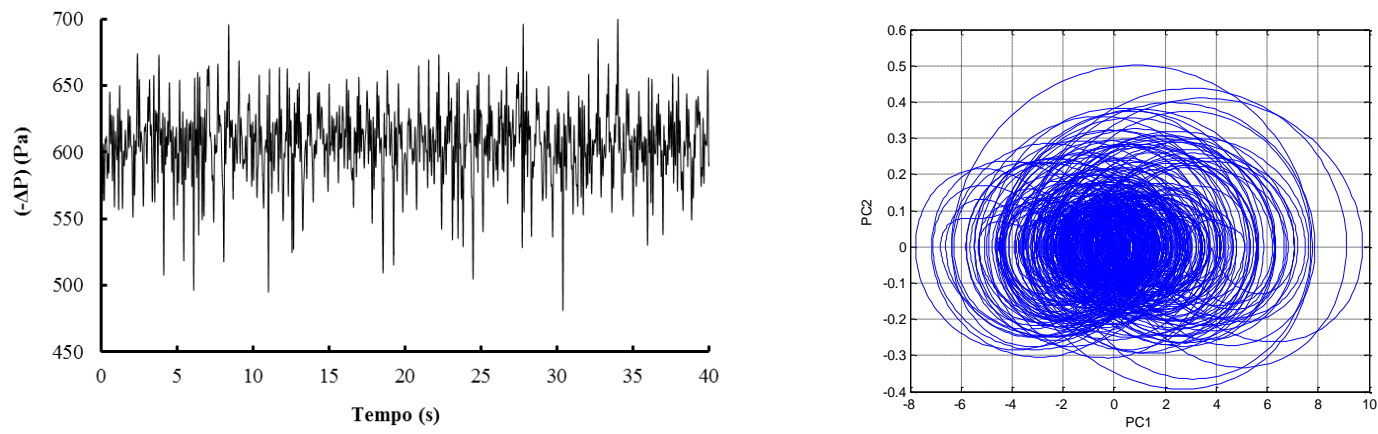

$\mathrm{U}=\mathbf{0 , 2 6} \mathrm{m} / \mathrm{s}$ (regime borbulhante)
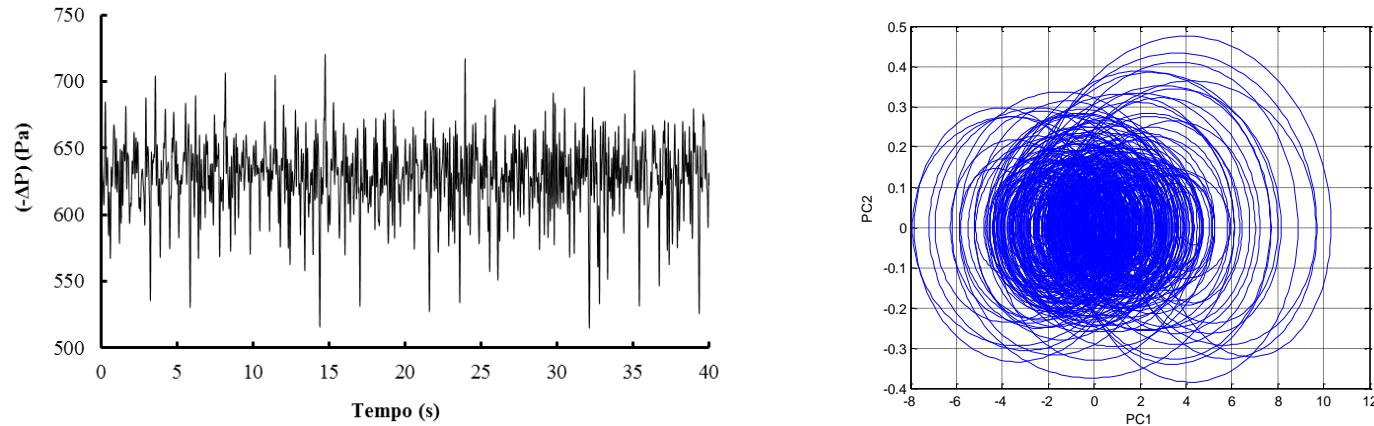

$\mathrm{U}=\mathbf{0 , 3 0} \mathrm{m} / \mathrm{s}$ (pistonado)
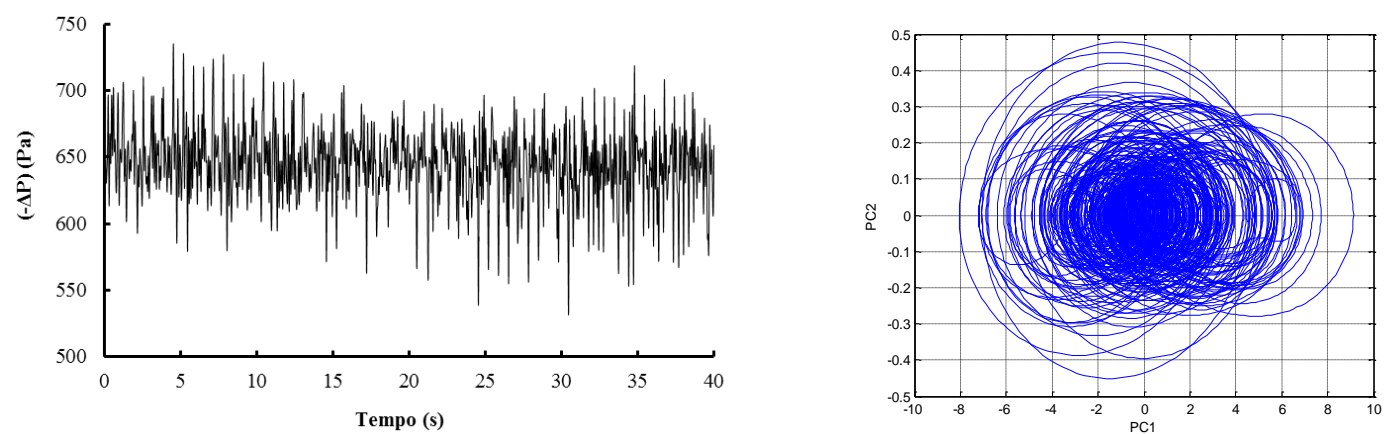
Esta dinâmica menos caótica faz com que o sistema perca menos informações e seja menos imprevisível em longo prazo, reduzindo a entropia de Kolmogorov. Outra constatação é que observando apenas curva fluidodinâmica não é possível notar a presença de uma região de transição, porém a análise do caos, muito mais sensível do que a clássica, exibe nitidamente esta faixa, tornando-se assim uma excelente ferramenta de análise de leitos fluidizados.

Figura 6 - Variação da entropia de Kolmogorov com o aumento da velocidade do gás - FCC.

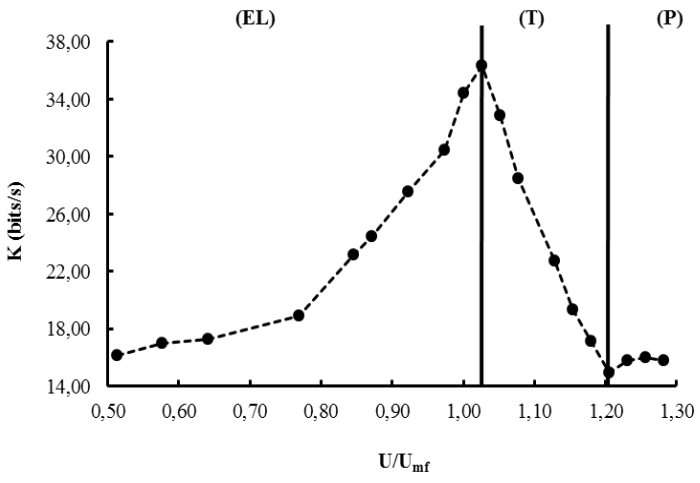

Figura 7 - Variação da entropia de Kolmogorov com o aumento da velocidade do gás - Microesferas de vidro.

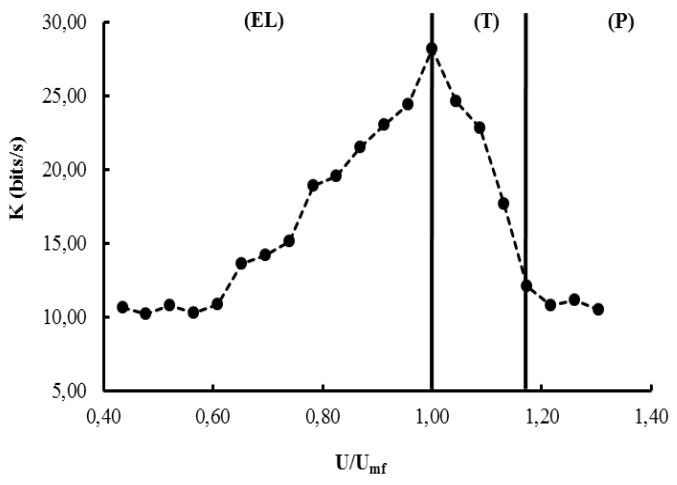

As variações dos valores da dimensão de correlação com a velocidade superficial do gás para o FCC e para as microesferas de vidro são apresentadas nas Figuras 8 e 9. Nas duas situações é possível observar efeito similar ao constatado para a entropia de Kolmogorov. Nota-se um acréscimo de D na medida em que a velocidade superficial do ar aumenta, alcançando seu valor máximo no momento em que o leito atinge a mínima fluidização e, logo em seguida, ocorre o decréscimo da complexidade do sistema quando as partículas começam a apresentar pistonamento.

Figura 8 - Variação da dimensão de correlação com o aumento da velocidade do gás - FCC.

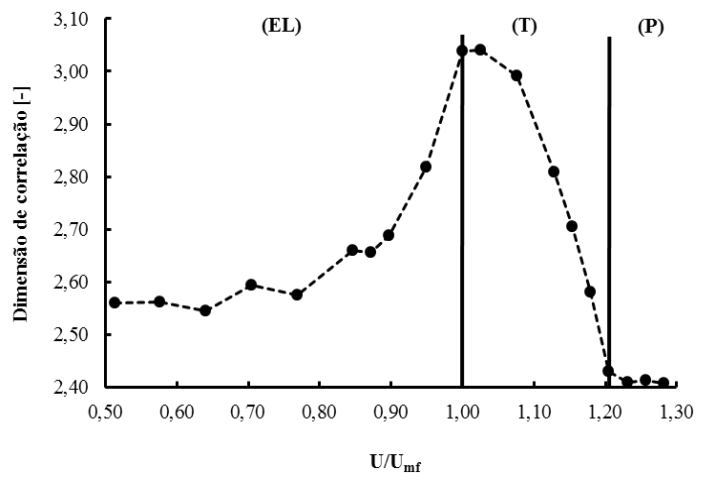

Figura 9 - Variação da dimensão de correlação com o aumento da velocidade do gás - Microesferas de vidro.

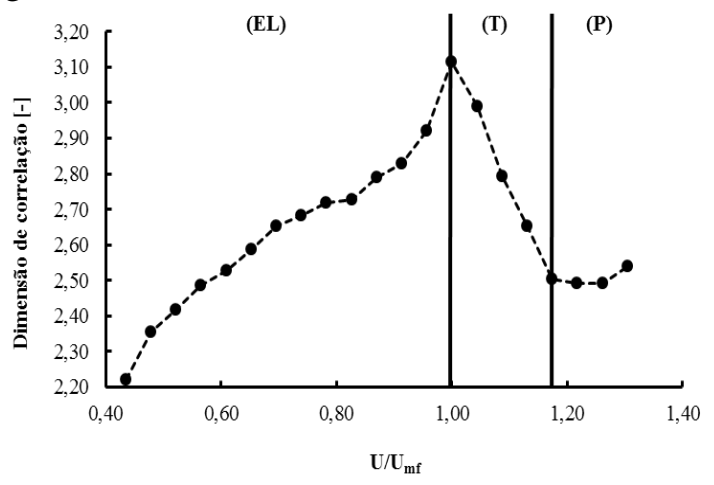

Trabalhos publicados por van den Bleek e Schouten (1993a, 1993b), Gou et al. (2003) e Llop et al (2012) também observaram declives, tanto na curva de $\mathrm{D}$ quanto $\mathrm{K}$, após atingida a fluidização. Esta constatação evidencia que a formação de bolhas aumenta a taxa de informação perdida e, na medida em que a velocidade do gás é incrementada, o regime pistonado é atingido, conferindo ao sistema características dinâmicas menos complexas. Esta fluidodinâmica menos 
caótica/complexa é observada até mesmo quando a turbulência é alcançada. Justificando esta informação, Llop et al. (2012) afirmam que a agitação vigorosa do leito faz com que ocorra uma estabilização e as estruturas formadas geram ordem.

Os baixos valores de dimensões de correlação ocorrem para velocidades do gás abaixo da mínima fluidização, e tendem a zero quando a velocidade também se aproxima de zero, apontando que as soluções temporais tendem a um ponto fixo. Transpondo a mínima fluidização, ocorre a presença de ciclo limite e o subsequente aumento de $D$ indica a presença de instabilidades geradas pelos choques entre partículas que, por sua vez, causam duplicação de período culminando em uma dinâmica caótica para as dimensões maiores que 2. Quando ocorre a transição de ambos os sistemas para o regime pistonado eles se tornam menos complexos gerando novo ciclo limite causando redução da dimensão de correlação.

\section{CONCLUSÕES}

Em termos de caracterização fluidodinâmica é possível e plausível utilizar os invariantes caóticos, pois, principalmente a entropia de Kolmogorov, exibiram relação direta com as faixas de transição dos regimes. Neste trabalho tornou-se evidente que a vigorosa agitação dos leitos, em regiões de altas velocidades, não necessariamente indica maior desorganização do sistema e que maior quantidade de informação é perdida quando da transição do regime borbulhante para pistonado.

Comparações entre os perfis caóticos de materiais pertencentes ao Grupo A de Geldart permitiram verificar que o parâmetro massa específica impactou decisivamente nos valores de entropia de Kolmogorov, apresentando maior desordem a fluidização de partículas mais pulverulentas. Para ambos os materiais, os perfis de entropia de Kolmogorov e de dimensão de correlação apresentaram picos característicos nas regiões de transição de leito fixo para mínima fluidização seguido de pistonado, deixando clara a relação entre esses parâmetros e os regimes de fluidização estudados. De mais a mais, a análise do caos possibilitou a identificação de regiões que a simples análise da curva fluidodinâmica não permitiria, demonstrando assim ser mais sensível do que a metodologia clássica.

Sendo assim, as nítidas diferenças apresentadas pelos invariantes para os diferentes regimes de fluidização e da evidente relação entre as características da fase particulada, considerando-se a classificação de Geldart, com os parâmetros caóticos, permitem corroborar tais invariantes como auxiliares na classificação dos regimes de fluidização.

\section{REFERÊNCIAS BIBLIOGRÁFICAS}

BAI, D.; GRACE, J. R.; ZHU, J. X. "Characterization of gas fluidized beds of group $\mathrm{C}, \mathrm{A}$ and $\mathrm{B}$ particles based on pressure fluctuations", Canadian Journal of Chemical Engineering, 77, p. 319, 1999.

BROWN, R.C.; Brue, E. "Resolving dynamical features of fluidized beds from pressure fluctuations", Powder Technology, 119, p. 68, 2001.

CASTILHO, G. J. "Análise de caos em leito fluidizado circulante". Tese (Doutorado em Engenharia Química), Faculdade de Engenharia Química, Universidade Estadual de Campinas, Campinas-SP, 2011.

\section{CREMASCO, M. A. Operações unitárias em sistemas particulados e} fluidomecânicos, São Paulo, Edgar Blucher, 2012. 
GOU, Q.; YUE, G.; SUDA, T.; SATO, J. "Flow characteristics in a bubbling fluidized bed at elevated temperature", Chem. Eng. Process.42, 439-447, 2003.

HONG, S. C.; JO, B. R.; DOH, D. S.; CHOI, C. S. "Determination of minimum fluidization velocity by the statistical analysis of pressure fluctuations in a gas-solid fluidized bed", Powder Technology, 60, p. 215, 1990.

JOHNSSON, F.; ZIJERVELD, R.C.; SCHOUTEN, J.C.; VAN DEN BLEEK, C.M., LECKNER, B. "Characterization of fluidization regimes by time-series analysis of pressure fluctuations", International Journal of Multiphase Flow, v. 26, p. 663-715, 2000.

KAGE, H.; IWASAKI, N.; YAMAGUCHI, H.; MATSUNO, Y. "Frequency analysis of pressure fluctuation in fluidized bed plenum", Journal of Chemical Engineering of Japan, 24, p. $76-81,1991$.

LLOP, M. F.; JANDB, N.; GALLUCCI, K.; LAURO, F. X. "Characterizing gas-solid fluidization by nonlinear tools: Chaotic invariants and dynamic moments", Chemical Engineering Science 71, 252-263, 2012.

MARTÍN, L.; BRIONGOS, J. V.; HERNANDO, G. N.; AARAGÓN, J. M. "Detecting regime transitions in gas-solid fluidized beds from low frequency accelerometry signals", Powder Technology, 207, p. 104 - 112, 2011.

MOURA, H. L. Análise da influência do ruído na obtenção de atratores e parâmetros determinísticos da teoria do caos aplicada em uma seção de riser de um leito fluidizado circulante. Faculdade de Engenharia Química, UNICAMP, Campinas-SP, 2013.

RUELLE, D.; TAKENS, F. "On the nature of turbulence", Commum. Math. Physics, v. 30, pp. 167-192, 1971.
SASIC, S.; LECKNER, B.; JOHNSSON, F. "Characterization of fluid dynamics of fluidized beds by analysis of pressure fluctuations", Progress in Energy and Combustion Science, 33, p. 453-496, 2007.

SAVI, M. A. "Dinâmica não linear e caos". Universidade Federal do Rio de Janeiro COPPE, Engenharia Mecânica: [s.n.], 2004.

STRINGER, J. "Is a fluidized bed a chaotic dynamic system", Proceedings of the 10th International Conference on Fluidized Bed Combustion, San Francisco, CA, v.1, pp. 265-272, 1989.

SUGIHARA, G., MAY, R. M.. Nonlinear forecasting as a way of distinguishing chaos from measurement error in time-series. Nature 344, 734 - 741. 1990.

TRNKA, O.; VESELÝ, V.; HARTMAN, M.; BERAN, Z. "Identification of the state of a fluidized bed by pressure fluctuation", AIChE Journal, 46, p. 509, 2000.

VAN DEN BLEEK, C. M.; SCHOUTEN, J. C. "Can Deterministic chaos create order in fluidized-bed scale-up?", Chemical Egineering Science, Vol. 48, No. 13, pp. 2367-2373, 1993.a.

VAN DEN BLEEK, C. M.; SCHOUTEN, J. C. "Deterministic chaos: a new tool in fluidized bed design and operation", Chemical Engineering Journal, v. 53, pp. 75-87, 1993.b.

YU, H-L.; LIN, Y-C.; SIVAKUMAR, B.; KUO, Y-M. A study of the temporal dynamics of ambient particulate matter using stochastic and chaotic techniques. Atmospheric Environment 69, 37-45, 2013. 